05,06

\title{
Магнитоэлектрический эффект в двухслойных композитах с градиентной магнитной фазой
}

\author{
(C) В.Н. Шут ${ }^{1}$, В.Л. Трубловский ${ }^{1}$, В.М. Лалетин ${ }^{1}$, И. Ядройцев ${ }^{2}$ \\ ${ }^{1}$ Институт технической акустики НАН Беларуси, \\ Витебск, Беларусь \\ ${ }^{2}$ Central University of Technology, \\ Free State, Bloemfontein, South Africa \\ E-mail: shut@vitebsk.by
}

Поступила в Редакцию 18 июня 2020 г.

В окончательной редакции 8 июля 2020 г.

Принята к публикации 9 июля 2020 г.

На основе твердых растворов никель-цинковых ферритов $\left(\mathrm{Ni}_{1-x} \mathrm{Zn}_{x}\right) \mathrm{Fe}_{2} \mathrm{O}_{4}$ изготовлены образцы однородной $(x=0 ; 0.1 ; 0.2)$ и многослойной керамики с градиентом состава $(x=0.2 \rightarrow 0.1 \rightarrow 0 \rightarrow 0.1 \rightarrow 0.2)$ по толстопленочной технологии. После спекания по двухступенчатому режиму градиентные образцы имели плавное неоднородное распределение химических элементов $(\mathrm{Zn}, \mathrm{Ni})$ по толщине.

Исследованы продольный $\left(\alpha_{E 33}\right)$ и поперечный $\left(\alpha_{E 31}\right)$ магнитоэлектрические эффекты в двухслойных композитах PZT-феррит никеля. Величины магнитоэлектрических коэффициентов в отсутствии внешнего постоянного магнитного поля были не значительны. Максимальное значение продольного магнитоэлектрического коэффициента в композитах с градиентной магнитной фазой практически в два раза превышало значение $\alpha_{E 33}$ в однородных структурах.

Ключевые слова: никель-цинковые ферриты, керамика с градиентом состава, магнитные свойства, магнитоэлектрический эффект.

DOI: $10.21883 /$ FTT.2020.11.50109.132

\section{1. Введение}

В последние годы сохраняется устойчивый интерес к магнитоэлектрикам (мультиферроикам) - классу материалов, в которых сосуществуют магнитное и электрическое упорядочение [1-3]. Взаимодействие электрической и магнитной подсистем в этих материалах проявляется в виде магнитоэлектрического (ME) эффекта. Этот эффект заключается в возникновении в образце электрического поля $\mathbf{E}$ (или поляризации $\mathbf{P}$ ) при приложении магнитного поля $\mathbf{H}\left(E=\alpha_{E} H-\right.$ прямой МЕ-эффект); или намагниченности $\mathbf{M}$ (или магнитного поля $\mathbf{H}$ ) при приложении электрического поля $E\left(H=\alpha_{H} E-\right.$ обратный МЕ-эффект). Наличие в материалах указанных эффектов открывает широкие перспективы их использования в различных приборах и устройствах, не связанных с протеканием постоянных электрических токов и сопряженных с этим тепловыми потерями $[4,5]$. Однако для однофазных магнитоэлектриков (таких как $\mathrm{BiFeO}_{3}$, $\mathrm{YMnO}_{3}, \mathrm{Cr}_{2} \mathrm{O}_{3}$ и др.) характерны малые величины МЕ-эффектов и низкие температуры их проявления, что не позволяло говорить об их практическом применении $[6,7]$. Ситуация стала коренным образом меняться после того, как была практически реализована идея создания композитных сред, состоящих из двух механически связанных друг с другом фаз: пьезомагнитной (или магнитострикционной) и пьезоэлектрической. Ключевыми моментами при создании композитов с большими ME-коэффициентами являются высокие характеристики отдельных компонентов, формирование качественного интерфейса (perfect interface) между магнитной и пьезоэлектрической фазами и тип связности $[1,3,8]$. Новым подходом, направленным на повышение свойств ME-композитов, является создание структур с градиентом состава (свойств), когда примеси распределены в объеме материала не однородно, а по некоторому закону. Теоретически предсказано, что МЕ-константы в слоистых композитах (со связностью 2-2) значительно возрастают при использовании структур с градиентом состава (свойств) в качестве сегнетоэлектрической и (или) магнитной компоненты [9]. В то же время, все применяемые в качестве магнитной фазы материалы (в частности, ферриты) не являются пьезомагнетиками. Их использование обусловлено наличием в этих материалах псевдопьезомагнитного эффекта $(q=d \lambda / d H$, $\lambda$ - коэффициент магнитострикции), проявляющегося при одновременном приложении к ним смещающего постоянного $\left(H_{d c}\right)$ и переменного $\left(H_{a c}\right)$ магнитных полей. В связи с этим, увеличение характеристик композитов может быть вызвано двумя факторами. Во-первых, в градиентных магнетиках теоретически предсказана возможность формирования внутреннего магнитного поля $H_{\text {int }}$, приводящего к сдвигу петли гистерезиса и появлению спонтанной намагниченности, направленной перпендикулярно плоскости образца [10]. В этом случае ME-эффект может проявляться без приложения смещающего постоянного поля. Во-вторых, при воздействии переменного магнитного поля $H_{a c}$ на материал с поперечной спонтанной намагниченностью, в магнитном слое возникают добавочные изгибные и крутящие 
моменты, способствующие увеличению электрического отклика [11].

Композиты, не требующие приложения внешних постоянных полей, получили название „самосмещенных“ ME-материалов (self biased magnetoelectric materials). Mandal и др. [11] исследовали двух$\left(\mathrm{PZT}-\mathrm{Ni}_{1-x} \mathrm{Zn}_{x} \mathrm{Fe}_{2} \mathrm{O}_{4}\right) \quad$ и трехслойные структуры $\left(\mathrm{PZT}-\mathrm{Ni}_{1-x} \mathrm{Zn}_{x} \mathrm{Fe}_{2} \mathrm{O}_{4}-\mathrm{PZT}\right)$. Градиент ферритового слоя формировался за счет последовательного соединения (склеивания) керамических дисков с различным соотношением Ni/Zn. Такие композиты характеризовались наличием ME-сигнала при $H_{d c}=0$ и увеличением его максимального значения при использовании градиентной магнитной подсистемы. Аналогичные результаты были получены и для других композитов с градиентной магнитной фазой [12-15]. Отметим, что полевые зависимости МЕ-коэффициента в указанных выше работах были симметричными.

Интересные результаты и иная их интерпретация были представлены Menghui Li и др. в [16]. В этой работе были получены двухслойные магнитные структуры путем склеивания аморфного Metglas и частично кристаллического Metglas. Аморфный и частично кристаллический Metglas имеют существенно различную магнитную „жесткость“ при близкой намагниченности насыщения. $M-H$-кривые для таких образцов были смещены по оси $H$, встроенное магнитное поле составляло $\left(H_{\text {bias }} \approx 2.7 \mathrm{Oe}\right)$. Такой сдвиг на кривой $M-H$ обусловлен магнитными взаимодействиями между „мягкой“ и „жесткой“ фазами. Симметричные композиты Metglas/PZT/Metglas на основе этих материалов обладали высоким МЕ-коэффициентом $\alpha_{E}=12 \mathrm{~V} \cdot \mathrm{cm}^{-1} \cdot \mathrm{Oe}^{-1}$ на частоте $f=1 \mathrm{kHz}$ при нулевом внешнем магнитном поле. Важно отметить, что полевые зависимости ME-коэффициента имели ярко выраженный несимметричный вид.

О наличии встроенного магнитного поля, проявляющегося в смещении $M-H$-кривых и не симметричных полевых зависимостях МЕ-коэффициента, сообщалось в $[17,18]$. В качестве пьезоэлектрика использовался AlN, в качестве магнитной компоненты - многослойные структуры $\mathrm{Ta} / \mathrm{Cu} / \mathrm{Mn}_{70} \mathrm{Ir}_{30} / \mathrm{Fe}_{50} \mathrm{Co}_{50}$ или $\mathrm{Ta} / \mathrm{Cu} / \mathrm{Mn}_{70} \mathrm{Ir}_{30} / \mathrm{Fe}_{70.2} \mathrm{Co}_{7.8} \mathrm{Si}_{12} \mathrm{~B}_{10}$, полученные ВЧ-магнетронным распылением. Возникновение $H_{\text {bias }}$ было обусловлено анизотропией обменного взаимодействия между антиферромагнетиком $\left(\mathrm{Mn}_{70} \mathrm{Ir}_{30}\right)$ и ферромагнетиком $\left(\mathrm{Fe}_{50} \mathrm{Co}_{50}\right)$. Не симметричная зависимость МЕ-коэффициента от внешнего поля наблюдалась в композитах, в которых градиентная магнитная фаза была получена с помощью электроосаждения различных металлов [19]. Более детально информация о „самосмещенных“ композитах систематизирована в [20].

Несмотря на многообещающие теоретические предсказания по поводу характеристик градиентных композитов, количество посвященных им публикаций невелико. В первую очередь это связано со сложностью практического получения композитов с изменяющимися в пространстве характеристиками (составом) даже одной из фаз - пьезоэлектрической или магнитной. Большинство проведенных к настоящему времени экспериментальных работ выполнены на структурах с небольшим числом слоев (обычно трех) в условиях, когда изменение параметров $q$ и $d$ является очень резким и происходит в узкой пространственной области на поверхности раздела фаз. Хотя эти работы в ряде случаев подтверждают теоретические предсказания, остается еще много непонятного и противоречивого в количественной оценке эффектов. Очевидно, что создание структур с плавным (в идеале непрерывным) изменением состава, исследование их интегральных характеристик, будет способствовать более глубокому пониманию отмеченных выше эффектов.

Целью настоящей работы является получение керамики на основе никель-цинковых ферритов $\left(\mathrm{Ni}_{1-x} \mathrm{Zn}_{x}\right) \mathrm{Fe}_{2} \mathrm{O}_{4}$ с градиентом состава, исследование ME-эффекта в двухслойных композитах пьезоэлектрикградиентный феррит.

\section{2. Образцы и методы эксперимента}

Порошки никель-цинковых ферритов были получены по керамической технологии с использованием оксидов $\mathrm{ZnO}$ и $\mathrm{Fe}_{2} \mathrm{O}_{3}$ марки ЧДА и $\mathrm{NiO}$ квалификации Ч $[21,22]$. Были синтезированы порошки $\left(\mathrm{Ni}_{1-x} \mathrm{Zn}_{x}\right) \mathrm{Fe}_{2} \mathrm{O}_{4}$ трех составов: $x=0 ; 0.1 ; 0.2$. Из приготовленных порошков методом шликерного литья получали пленки толщиной $\sim 27-28 \mu \mathrm{m}$. Однородные структуры (с фиксированной концентрацией цинка) собирались из пленок одинакового состава. Для изготовления структур с градиентом состава $(x=0.2 \rightarrow 0 \rightarrow 0.2)$ совместно прессовались пленки различных составов $\quad\left(\left(\mathrm{Ni}_{0.8} \mathrm{Zn}_{0.2}\right) \mathrm{Fe}_{2} \mathrm{O}_{4}+\left(\mathrm{Ni}_{0.9} \mathrm{Zn}_{0.1}\right) \mathrm{Fe}_{2} \mathrm{O}_{4}+\mathrm{NiFe}_{2} \mathrm{O}_{4}+\right.$ $\left.+\left(\mathrm{Ni}_{0.9} \mathrm{Zn}_{0.1}\right) \mathrm{Fe}_{2} \mathrm{O}_{4}+\left(\mathrm{Ni}_{0.8} \mathrm{Zn}_{0.2}\right) \mathrm{Fe}_{2} \mathrm{O}_{4}\right)$ - по два слоя каждого состава (рис. 1).

Из пакетов вырубались заготовки размерами $5.5 \times 4.0 \mathrm{~mm}$. Для уменьшения диффузионных процессов на границах слоев с различным составом был применен двухступенчатый режим спекания образцов. На первом этапе температура повышалась до максимального значения $1270^{\circ} \mathrm{C}$ (скорость нагрева составляла

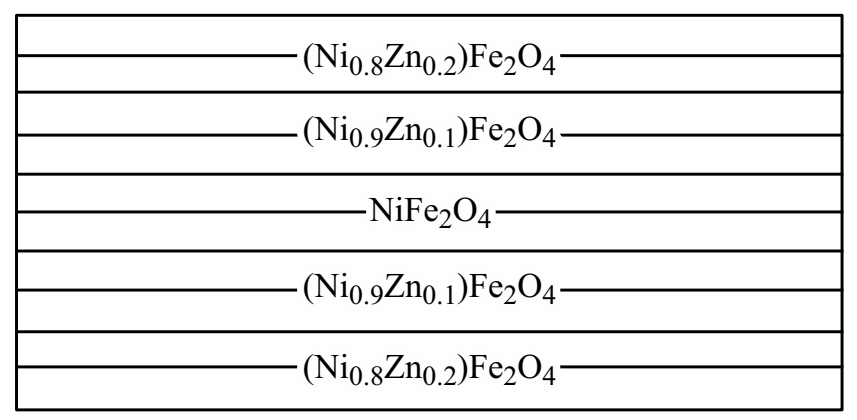

Рис. 1. Схема структуры с „встречным“ градиентом состава $(x=0.2 \rightarrow 0 \rightarrow 0.2)$. 


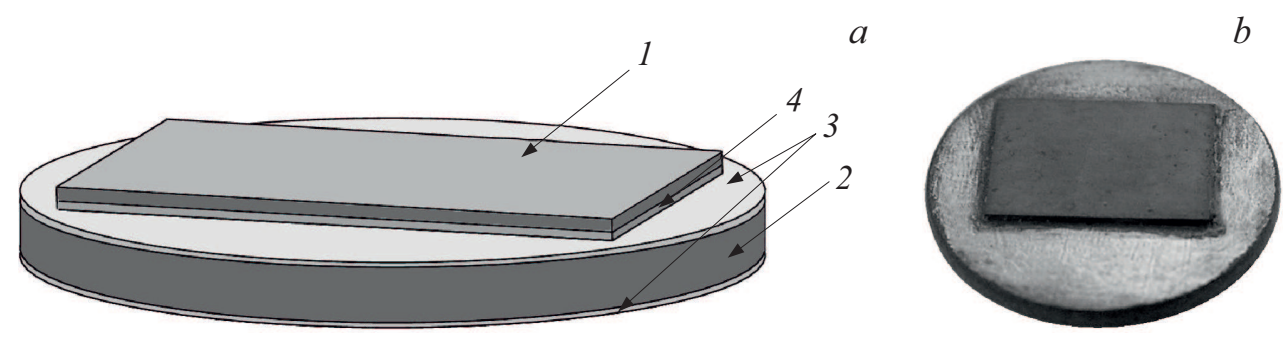

Рис. 2. Схема структуры и фотография МЕ-композита: $1-\left(\mathrm{Ni}_{1-x} \mathrm{Zn}_{x}\right) \mathrm{Fe}_{2} \mathrm{O}_{4}, 2-\mathrm{PZT}, 3-$ серебряные электроды, $4-$ клей.
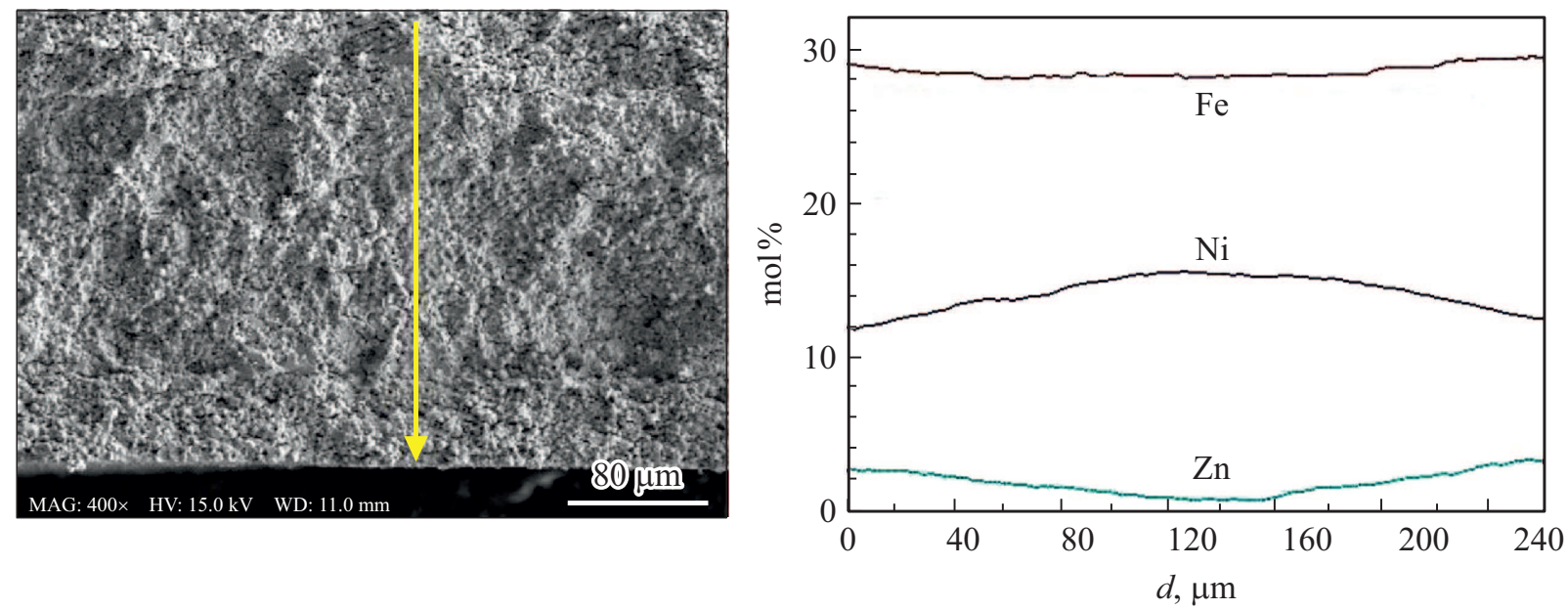

Рис. 3. Фотография торца (стрелкой указана линия, вдоль которой проводились измерения) и распределение элементов по толщине градиентной керамики $\left(\mathrm{Ni}_{1-x} \mathrm{Zn}_{x}\right) \mathrm{Fe}_{2} \mathrm{O}_{4}(x=0.2 \rightarrow 0 \rightarrow 0.2)$.

$350^{\circ} \mathrm{C} / \mathrm{h}$ ), затем снижалась до $1100^{\circ} \mathrm{C}$, при которой осуществлялась выдержка в течение $3 \mathrm{~h}$. Окончательная толщина многослойных образцов составляла $\sim 260 \mu \mathrm{m}$. Полученные керамические образцы не имели видимых деформационных искажений.

Для приведения всех образцов к одинаковой толщине и обеспечения плоскостности поверхности, однородная и градиентная магнитная керамика шлифовалась с одной стороны до толщины $180 \mu \mathrm{m}$. Кроме того, в случае градиентных образцов эта операция необходима для создания однонаправленного градиента состава. В качестве пьезоэлектрика использовалась керамические диски PZT диаметром 8.9 и толщиной $0.62 \mathrm{~mm}$. Таблетки покрывались серебряными электродами и затем поляризовались в постоянном электрическом поле. Для формирования композита пьезозоэлектрические и магнитные материалы склеивались в соответствии со схемой, приведенной на рис. 2.

Исследования микроструктуры и распределения химических элементов проводились на сканирующем электронном микроскопе высокого разрешения S-4800 (Hitachi) с рентгеновским спектрометром QUANTAX 200.

Магнитоэлектрический сигнал измерялся при двух вариантах эксперимента: поперечный (transverse) MEкоэффициент $\alpha_{E 31}$ определялся, когда постоянное $H_{d c}$ и переменное $H_{a c}$ магнитные поля были параллельны друг другу и главной плоскости образцов (перпендикулярны $P_{S}$; п продольный (longitudinal) ME-коэффициент $\alpha_{E 33}$ определялся, когда постоянное и переменное магнитные поля были параллельны друг другу и перпендикулярны плоскости образцов (параллельны $P_{S}$ ).

\section{3. Результаты и их обсуждение}

Результаты исследований структуры и свойств никельцинковых ферритов $\left(\mathrm{Ni}_{1-x} \mathrm{Zn}_{x}\right) \mathrm{Fe}_{2} \mathrm{O}_{4}$ подробно описаны в работах $[21,22]$. Все полученные магнитные материалы имели шпинельную структуру. Параметры элементарной ячейки увеличивались при увеличении концентрации цинка $(a=8.34 \AA$ при $x=0, a=8.40 \AA$ при $x=0.2)$. Образцы имели однородную микроструктуру, размеры зерен составлял $2-5 \mu \mathrm{m}$ (и незначительно изменялся с изменением концентрации цинка). Зависимости удельной намагниченности от величины индукции магнитного поля $\sigma=f(B)$ были изучены при комнатных температурах в геометрии параллельного и перпендикулярного направления магнитного поля относительно плоскости пленок. В однородных образцах удельная намагниченность увеличивалась от $\sigma=46 \mathrm{~A} \cdot \mathrm{m}^{2} \cdot \mathrm{kg}^{-1}$ (при $\left.x=0\right)$ до $\sigma=67 \mathrm{~A} \cdot \mathrm{m}^{2} \cdot \mathrm{kg}^{-1}$ (при $\left.x=0.2\right)$. Петли магнитного гистерезиса градиентных материалов были симметричными по обеим осям. Искажений в виде перетяжек также не наблюдалось. 

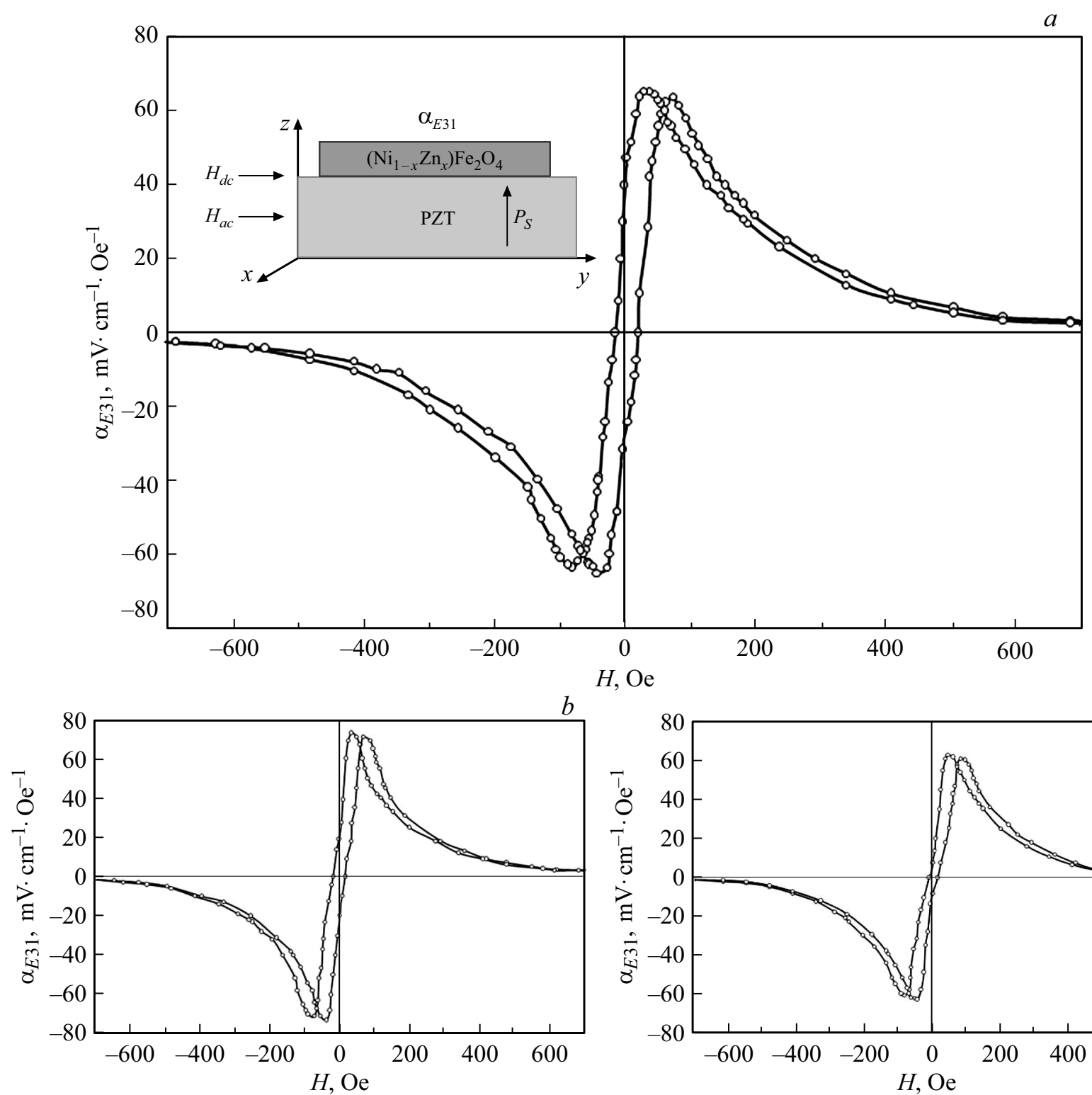

(

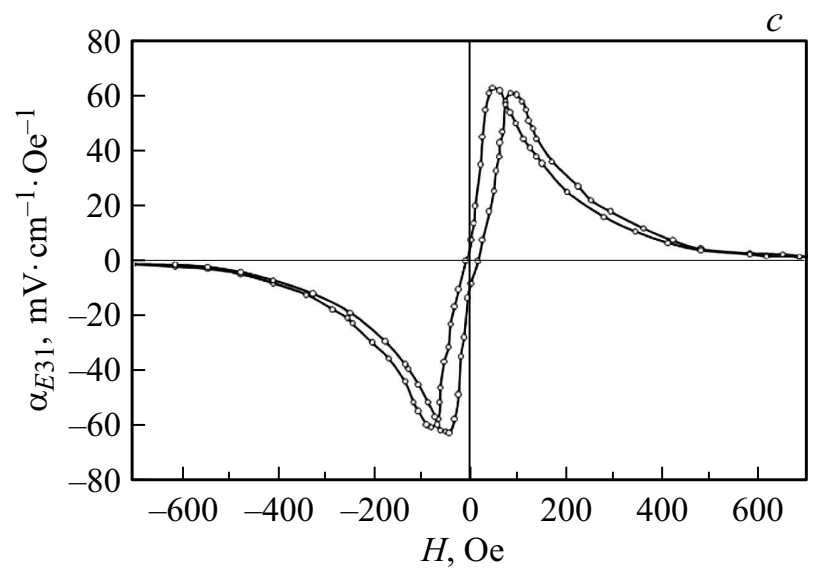

Pис. 4. Полевые зависимости поперечного МЕ-коэффициента $\alpha_{E 31}$ при различном составе магнитной фазы: $\left.a\right) \mathrm{NiFe}_{2} \mathrm{O}_{4}$, b) $\left.\left(\mathrm{Ni}_{0.8} \mathrm{Zn}_{0.2}\right) \mathrm{Fe}_{2} \mathrm{O}_{4}, c\right)$ градиентный феррит.

Поперечный МЕ-эффект в двухслойных структурах пьезоэлектрик- $-\left(\mathbf{N i}_{1-x} \mathbf{Z n}_{x}\right) \mathbf{F e}_{2} \mathbf{O}_{4}$. Отметим, что изначально были изготовлены структуры магнитных материалов $\left(\mathrm{Ni}_{1-x} \mathrm{Zn}_{x}\right) \mathrm{Fe}_{2} \mathrm{O}_{4}$ со ступенчатым изменением состава $(x=0.2 \rightarrow 0.1 \rightarrow 0 \rightarrow 0.1 \rightarrow 0.2)$. После спекания концентрация цинка плавно изменялась от $\sim 0.3 \mathrm{~mol} . \%$ в центре до $\sim 2.3 \mathrm{~mol} . \%$ на краю образцов (рис. 3 ). Концентрация никеля составляла $13 \mathrm{~mol} \%$ в центре и $10.9 \mathrm{~mol} \%$ на краю (при общей толщине $\sim 260 \mu \mathrm{m}$ ) [22]. Таким образом, используемый двухстадийный режим спекания позволяет достичь компромисса: обеспечить синтез керамики и сохранить при этом закономернонеоднородное распределение химических элементов. Далее образцы шлифовались до толщины $180 \mu \mathrm{m}$. Поэтому в градиентных ферритах, используемых при изготовле- нии магнитоэлектрических композитов, изменение концентрации цинка по толщине составляло $0.3-2.3 \mathrm{~mol} . \%$.

Полевые зависимости поперечного МЕ-коэффициента $\alpha_{E 31}$ (магнитное поле направлено перпендикулярно оси поляризации) приведены на рис. 4. Они имеют типичный вид - с увеличением постоянного магнитного поля МЕ-коэффициент увеличивается, достигает максимума и затем снижается до нуля. МЕ-сигнал прямо пропорционален псевдопьезомагнитному коэффициенту $q=d \lambda / d H$ и, следовательно, $H$-зависимость по существу повторяет наклон $\lambda$ от $H$. Для соединения феррита никеля цинка теоретически рассчитано, что $q$ линейно возрастает с увеличением концентрации цинка в диапазоне $x=0-0.3$, а затем снижается при $x>0.3$. Экспериментальные исследования двухслойных 


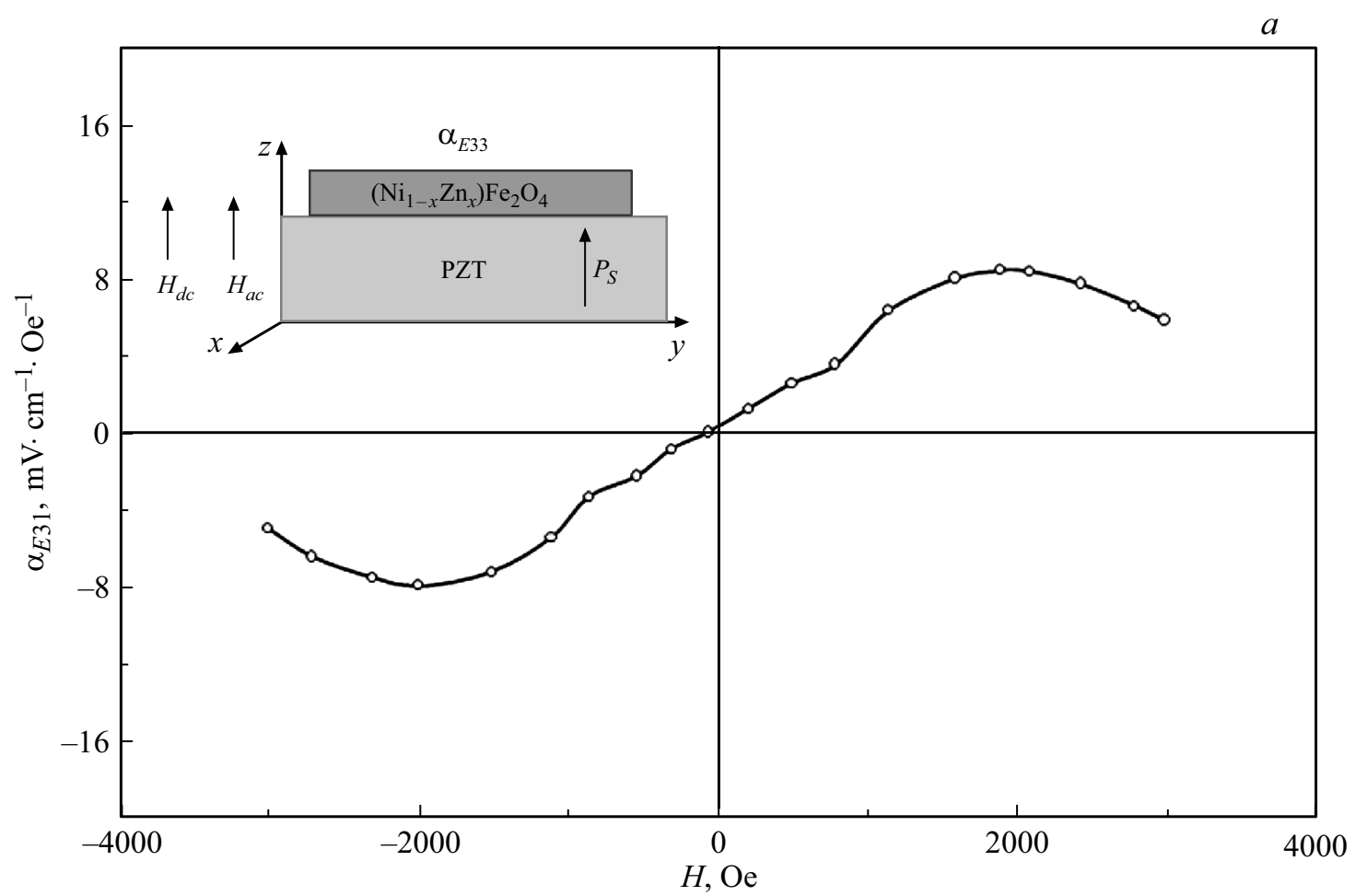

$b$
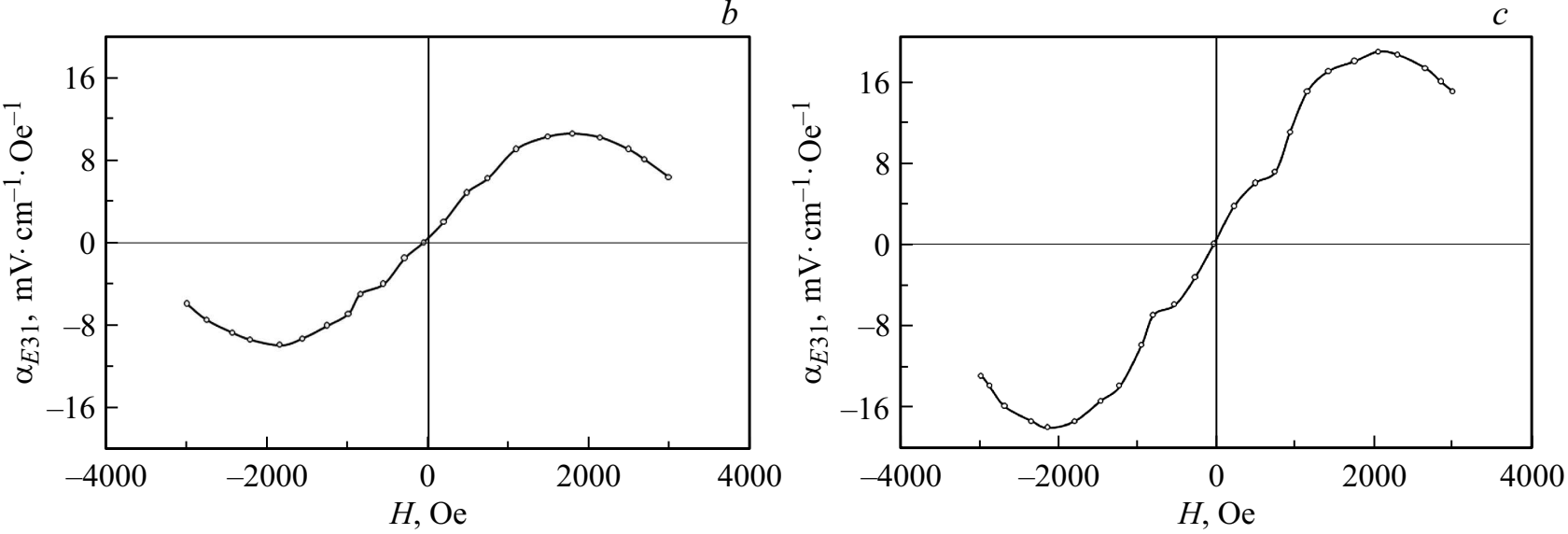

Рис. 5. Полевые зависимости продольного МЕ-коэффициента $\alpha_{E 33}$ при различном составе магнитной фазы: $a$ ) $\mathrm{NiFe}_{2} \mathrm{O}_{4}$, b) $\left.\left(\mathrm{Ni}_{0.8} \mathrm{Zn}_{0.2}\right) \mathrm{Fe}_{2} \mathrm{O}_{4}, c\right)$ градиентный феррит.

композитов в целом подтверждают эту тенденцию [23]. Однако для керамической технологии характерны достаточно большие разбросы характеристик. Они могут быть обусловлены отклонением свойств пьезоэлектрической и магнитоэлектрической фазы, а также различным коэффициентом связи на границе слоев структуры в различных образцах. В наших экспериментах магнитоэлектрический коэффициент возрастал при увеличении концентрации цинка $\left(\alpha_{E 31}=65.4 \mathrm{mV} \cdot \mathrm{cm}^{-1} \mathrm{Oe}^{-1}\right.$ при $x=0 ; \quad \alpha_{E 31}=73.1 \mathrm{mV} \cdot \mathrm{cm}^{-1} \mathrm{Oe}^{-1}$ при $\left.x=0.2\right)$. При замещении однородного феррита на градиентный МЕсигнал был несколько ниже: $\alpha_{E 31}=63.6 \mathrm{mV} \cdot \mathrm{cm}^{-1} \mathrm{Oe}^{-1}$. В двухслойных структурах пьезомагнитный коэффициент определяется как $q=q_{11}+q_{12}=d \lambda_{11} / d H$ $+d \lambda_{12} / d H$, где $\lambda_{11}$ - коэффициент магнитострикции в направлении, параллельном магнитному полю $H, \lambda_{12}-$ перпендикулярно $H$. В двухслойных структурах внешнее магнитное поле генерирует два типа механических напряжений: продольные напряжения (in-plane strain), вызванные магнитострикцией, и напряжения, обусловленные деформацией изгиба из-за асимметрии структуры. Изгибные напряжения имеют составляющую в направлении продольных напряжений (in-plane component) и уменьшают общую интенсивность МЕ-взаимодействия [11]. При создании композитов с градиентной магнитной фазой возможны два варианта формирования структуры: отрицательный градиент $q$, когда величина $q$ уменьшается от интерфейса PZT - феррит; и положительный градиент. Во втором случае, положительный градиент $q$ способствует появлению изгибающего 
момента, направленного противоположно деформации изгиба, вызванной асимметрией структуры. В результате ME-коэффициент возрастает. В наших экспериментах двухслойный композит имеет положительный градиент $q$. Однако МЕ-коэффициент не увеличивается. Этот факт можно объяснить зависимостью интенсивности МЕ-взаимодействия от объемной доли феррита по отношению к пьезоэлектрику. Наиболее сильно влияние градиентной структуры проявляется, когда объемная доля феррита составляет более 20-30\%. Когда объемная доля феррита меньше $20 \%$ МЕ-коэффициент резко уменьшается и его величина не зависит от того, является ли магнитная подсистема однородной или градиентной (а также не зависит от направления градиента $q$ ) [9]. В наших образцах объемное соотношение феррит/PZT составляло $\sim 0.12$.

Продольный МЕ-эффект в двухслойных структуpax пьезоэлектрик- $\left(\mathrm{Ni}_{1-x} \mathbf{Z n}_{x}\right) \mathbf{F e}_{2} \mathbf{O}_{4}$. При исследовании продольного ME-эффекта $\alpha_{E 33}$ внешнее постоянное и переменное магнитные поля были направлены параллельно вектору спонтанной поляризации PZT и градиенту намагниченности (или градиенту состава). Именно такое расположение внешнего поля относительно направления градиента намагниченности (состава) наиболее благоприятно для возникновения в системе новых эффектов. Как было отмечено, в наших градиентных ферритах сдвига и искажений петель гистерезиса обнаружено не было. Это свидетельствует об отсутствии внутреннего магнитного поля, либо о малой величине $H_{\text {int }}$. Поэтому величина МЕ-коэффициента в отсутствии внешнего магнитного поля незначительна (рис. 5). В двухслойных композитах небольшие МЕ-сигналы могут проявляться из-за несимметричности структур и гистерезисных явлений. Полевые зависимости $\alpha_{E 33}$ практически симметричны, что также подтверждает отсутствие внутреннего магнитного поля в ферритах.

Максимальное значение МЕ-коэффициента существенно возрастает при использовании градиентных материалов в качестве магнитной подсистемы по сравнению с однородными структурами $\left(\alpha_{E 33}=18.9 \mathrm{mV} \cdot \mathrm{cm}^{-1} \mathrm{Oe}^{-1}\right.$ для градиентной структуры; $\alpha_{E 33}=8.4 \mathrm{mV} \cdot \mathrm{cm}^{-1} \mathrm{Oe}^{-1}$ для однородного образца c $x=0 ; \alpha_{E 33}=10.5 \mathrm{mV} \cdot \mathrm{cm}^{-1} \mathrm{Oe}^{-1}$ для однородного образца с $x=0.2)$. Здесь следует подчеркнуть, что продольный и поперечный МЕ-эффекты были исследованы на одних и тех же композитах, т.е. при двух геометриях эксперимента магнитные, пьезоэлектрические, механические свойства материалов, а также коэффициент связи между слоями были идентичны. Следовательно, увеличение продольного МЕ-коэффициента в градиентных структурах имеет место при отсутствии внутреннего магнитного поля. Наши результаты не позволяют утверждать, что повышение величины $\alpha_{E 33}$ обусловлены только наличием градиента намагниченности. Возможно, в таких композитах возникают дополнительные механические напряжения благодаря наличию градиента упру- гих свойств, обусловленных закономерно неоднородным изменением состава.

Как правило, в композитах (в том числе и градиентных) исследуется поперечный сигнал. Это связано с тем, что его величина более чем в два раза больше по сравнению с продольным сигналом $\left(\alpha_{E 31}>2 \alpha_{E 33}\right)$, и он проявляется при меньших внешних магнитных полях. О многократном увеличении $\alpha_{E 33}$ сообщалось в [2]. В этой работе приведены результаты исследования симметричной структуры PZT-compositionally graded $\mathrm{Ni}_{1-x} \mathrm{Zn}_{x} \mathrm{Fe}_{2} \mathrm{O}_{4}-\mathrm{PZT}$. При замещении однородного феррита никеля на градиентный, максимальное значение $\alpha_{E 33}$ увеличивалось более чем в 10 раз. При этом композиты не проявляли значительного МЕ-взаимодействия в отсутствии внешнего смещающего поля $H_{d c}$, что находится в соответствии с полученными нами результатами.

\section{4. Заключение}

По толстопленочной технологии получены образцы однородной $(x=0 ; 0.1 ; 0.2)$ и многослойной керамики с градиентом состава на основе твердых растворов никель-цинковых ферритов $\left(\mathrm{Ni}_{1-x} \mathrm{Zn}_{x}\right) \mathrm{Fe}_{2} \mathrm{O}_{4}$. Изменение концентрации цинка по толщине градиентных образцов составляло $0.3-2.3 \mathrm{~mol} \%$. Изготовлены двухслойные композиты $\mathrm{PZT}-\mathrm{NiFe}_{2} \mathrm{O}_{4}, \mathrm{PZT}-\left(\mathrm{Ni}_{0.8} \mathrm{Zn}_{0.2}\right) \mathrm{Fe}_{2} \mathrm{O}_{4}$, $\mathrm{PZT}$-градиентный $\left(\mathrm{Ni}_{1-x} \mathrm{Zn}_{x}\right) \mathrm{Fe}_{2} \mathrm{O}_{4}$. Исследованы продольный и поперечный МЕ-эффекты в полученных композитах. Величины МЕ-коэффициентов в отсутствии внешнего магнитного поля были незначительны. Максимальное значение поперечного МЕ-коэффициента не увеличивалось при замене однородного феррита на градиентный феррит. Максимальное значение продольного МЕ-коэффициента практически в два раза увеличивалось в композитах с градиентной магнитной фазой по сравнению с однородными структурами $\left(\alpha_{E 33}=18.9 \mathrm{mV} \cdot \mathrm{cm}^{-1} \mathrm{Oe}^{-1}\right.$ для градиентной структуры; $\alpha_{E 33}=8.4 \mathrm{mV} \cdot \mathrm{cm}^{-1} \mathrm{Oe}^{-1}$ для однородного образца c $x=0 ; \quad \alpha_{E 33}=10.5 \mathrm{mV} \cdot \mathrm{cm}^{-1} \mathrm{Oe}^{-1}$ для однородного образца с $x=0.2)$. Полевые зависимости $\alpha_{E 33}$ практически симметричны, что свидетельствует об отсутствии внутреннего магнитного поля в ферритах. Возможно, в композитах возникают дополнительные механические напряжения благодаря наличию градиента упругих свойств, обусловленных закономерно неоднородным изменением состава.

\section{Финансирование работы}

Работа выполнена при частичной поддержке Белорусского фонда фундаментальных исследований (грант № Ф20MC-026).

\section{Конфликт интересов}

Авторы заявляют, что у них нет конфликта интересов. 


\section{Список литературы}

[1] J. Ma, J. Hu, Z. Li, C.-W. Nan. Adv. Mater. 23, 1062 (2011).

[2] G. Srinivasan. Annu. Rev. Mater. Res. 40, 153 (2010).

[3] C.-W. Nan, M.I. Bichurin, S. Dong, D. Viehland, G. Srinivasan. J. Appl. Phys. 103, 031101 (2008).

[4] C.M. Leung, J.-F. Li, D. Viehland, X. Zhuang. J. Phys. D 51, 263002 (2018)

[5] J.-M. Hu, L.-Q. Chen, C.-W. Nan. Adv. Mater. 28, 15 (2016).

[6] T. Jia, Z. Cheng, H. Zhao, H. Kimura. Appl. Phys. Rev. 5, 021102 (2018).

[7] A.N. Masyugin, S.S. Aplesnin, Y.Y. Loginov, O.N. Bandurina. IOP Conf. Ser: Mater. Sci. Eng. 822, 012025 (2020).

[8] В.Н. Шут, В.М. Лалетин, С.Р. Сырцов, В.Л. Трубловский, Ю.В. Медведева, К.И. Янушкевич, М.В. Бушинский, Т.В. Петлицкая. ФТТ 60, 1699 (2018).

[9] V.M. Petrov, G. Srinivasan. Phys. Rev. B 78, 184421 (2008).

[10] J.V. Mantese, A.L. Micheli, N.W. Schubring, R.W. Hayes, G. Srinivasan, S.P. Alpay. Appl. Phys. Lett. 87, 082503 (2005).

[11] S.K. Mandal, G. Sreenivasulu, V.M. Petrov, G. Srinivasan. Appl. Phys. Lett. 96, 192502 (2010).

[12] S.K. Mandal, G. Sreenivasulu, V.M. Petrov, G. Srinivasan. Phys. Rev. B 84, 014432 (2011).

[13] G. Sreenivasulu, S.K. Mandal, S. Bandekar, V.M. Petrov, G. Srinivasan. Phys. Rev. B 84, 144426 (2011).

[14] U. Laletin, G. Sreenivasulu, V.M. Petrov, T. Garg, A.R. Kulkarni, N. Venkataramani, G. Srinivasan. Phis. Rev. B 85, 104404 (2012).

[15] S.-C. Yang, C.-S. Park, K.-H. Cho, S.J. Priya. J. Appl. Phys. 108, 093706 (2010).

[16] M. Li, Z. Wang, Y. Wang, J. Li, D. Viehland. Appl. Phys. Lett. 102, 082404 (2013).

[17] E. Lage, C. Kirchhof, V. Hrkac, L. Kienle, R. Jahns, R. Knöchel, E. Quandt, D. Meyners. Nature Mater. 11, 523 (2012).

[18] E. Lage, N.O. Urs, V. Röbisch, I. Teliban, R. Knöchel, D. Meyners, J. Mc Cord, E. Quandt. Appl. Phys. Lett. 104, 132405 (2014).

[19] D. Xie, Y.G. Wang, J.H. Cheng. J. Mater. Sci.: Mater. Electron. 26, 3545 (2015).

[20] Y. Zhou, D. Maurya, Y. Yan, G. Srinivasan, E. Quandt, S. Priya. Energy Harvest. Syst. 3, 1 (2016).

[21] В.Н. Шут, С.Р. Сырцов, Л.С. Лобановский, К.И. Янушкевич. ФТТ 58, 1907 (2016).

[22] В.Н. Шут, С.Р. Сырцов, В.Л. Трубловский, М. Vijatovic Petrovic. ФTT 61, 1793 (2019).

[23] G. Srinivasan, V.M. Laletsin, R. Hayes, N. Puddubnaya, E.T. Rasmussen, D.J. Fekel. Solid State Commun. 124, 373 (2002).

Редактор Т.Н. Василевская 